\title{
Remote monitoring of medical devices in Australia
}

\author{
The current industry-involved system may present a conflict of interest
}

$\mathrm{M}$ ore patients, and therefore medical professionals at all levels, are encountering devices that are being remotely monitored. While all currently implanted pacemakers and defibrillators are now remote-monitoring capable, there has also been a substantial increase in the use of implantable loop monitors - from 987 in the 2013-14 financial year to 2269 in 2015-16 (Medicare Benefits Schedule item 38285; http://medicarestatistics. humanservices.gov.au/statistics/mbs_item.jsp). This increase has forced the health care system to engage with remote monitoring.

There is accumulating evidence supporting remote interrogation and monitoring for cardiovascular implantable electronic devices. ${ }^{1-3}$ As a result, remote monitoring has become the standard of care and is reflected in a 2015 consensus statement developed in collaboration with, and endorsed by the Asia Pacific Heart Rhythm Society and American, European and Latin American societies. ${ }^{4}$

The consensus statement clearly advises that industry "should refrain from direct patient care, either within the clinic or at home" and "should not perform, collect, or triage data". ${ }^{5}$ However, there are significant international geographical differences in the ways in which remote monitoring is reimbursed, which affect the use of these guidelines in Australia.

Since 2013, when Lau and Zhang ${ }^{5}$ discussed remote monitoring in the Asia-Pacific region, the reimbursement model has changed. Within the private system in Australia, the current physician reimbursement for a year of remotely monitoring a pacemaker is $\$ 50.15$ ( $75 \%$ of the $\$ 66.85$ scheduled fee), and $\$ 142.15$ (75\% of the $\$ 189.50$ scheduled fee) for defibrillators. ${ }^{6}$ Implantable loop recorders are not reimbursed for remote monitoring. In Australia, the current rebate provided to industry for establishing remote monitoring in the private system is $\$ 1960$ (as of November 2016). ${ }^{7}$ At present, this includes only the cost of providing the remote monitor (and the associated information technology infrastructure to permit the use of a remote system). The device itself costs about $\$ 500$, with no commitment to follow the device. Independently of whether such reimbursements are appropriate, they are currently in place, very unlikely to change in the near future, and unique to Australia.
Bradley Wilsmore

James Leitch

John Hunter Hospital, Newcastle, NSW. bwilsmore@ gmail.com
The reimbursement for remote monitoring is not provided in the public system, and therefore there has not been the same enthusiasm from industry to provide a service for remote monitoring publicly, which would be expected to reduce the benefits obtained by remote monitoring. However, remote monitoring has been shown to reduce overall health expenditure ${ }^{8}$ by reducing staffing and time in clinics, even in a non-reimbursed

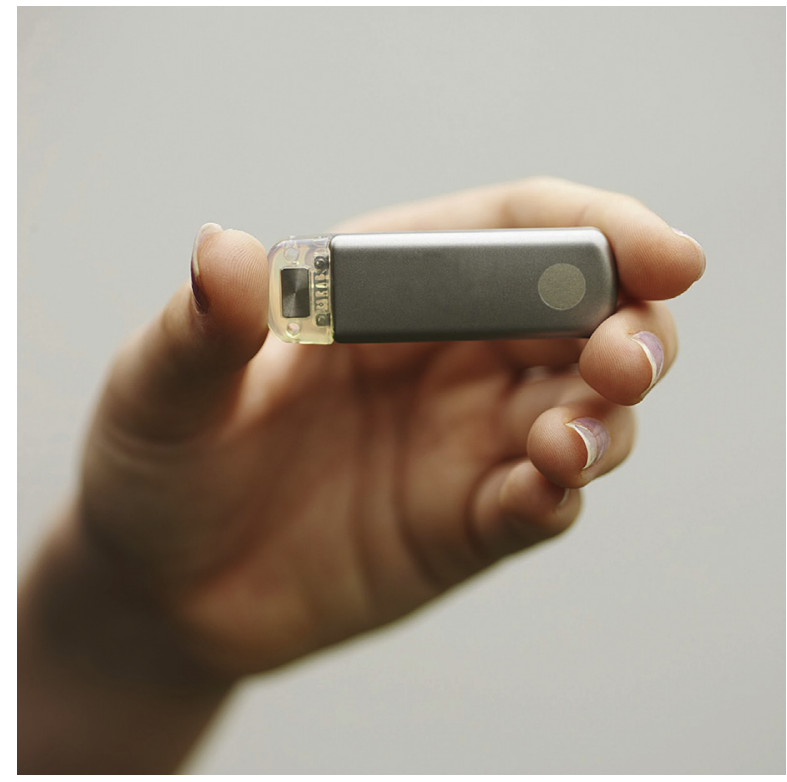

administrators may be more inclined to support an infrastructure for remote monitoring even in the absence of receiving a private prosthesis reimbursement.

As a result of an environment where a physician is not reimbursed for a full year of remotely monitoring an implantable loop monitor, and where the industry receives $\$ 1960$ (about $\$ 1500$ more than the remote monitoring component is worth) in addition to the cost of the actual loop monitor, some of the time and financial commitments are already shifting to industry. The device industries in Australia are now increasingly willing to fund a system where all remote monitoring occurs via their own trained industry representatives, who will generally educate the patient at implant, may contact the patient directly at home to facilitate the set-up and transmission process along with troubleshooting, then triage and report on the transmitted data, before providing this information to the physician. This is all being facilitated at no charge to the physician but utilises the government-funded, private prosthesis reimbursement at implant (\$1960) paid to the industry. This clearly conflicts with the current guidelines. The primary limitation to this industry-involved system relates to a conflict of interest. The ultimate responsibility of for-profit entities is to maximise returns to shareholders. In contrast, the medical profession should put patients' interests first, regardless of financial considerations.

A company's willingness to provide this service is likely to be multifactorial. From a business perspective, in a highly competitive device environment, providing a service which relieves physician time and financial commitments may increase a particular company's market share, and the unique distribution of funding provided by the 
government has allowed companies to fund such an infrastructure. Additionally, it is more economical than sending field representatives to perform in-person checks. Further, all companies have an inherent desire to provide a service to their patients that improves patient care. Given the funding arrangements, and the idiosyncrasies of each of the five major device companies regarding device-related features and remote monitoring set-up, there are some clear advantages to this model.

Setting up an infrastructure to manage remote monitoring involves managing remote transmissions, which occur 24 hours a day, 365 days a year. This raises medico-legal issues relating to both a guideline recommendation that not providing remote monitoring may risk liability, and if it is provided, a burden of care to provide appropriate responses within a reasonable time frame. All companies have acknowledged the physician as ultimately medicolegally responsible for the patient's care. It is unlikely that remote monitoring would consistently delay feedback on clinically relevant information relative to the current standard of care, which generally involves 6-monthly in-person device checks; however, with remote monitoring comes an expectation of much more rapid feedback. Addressing these expectations upfront before initiating monitoring and documentation is likely to minimise the risk of medico-legal consequences. While there are also non-device-associated third party providers offering a similar service, this would not entirely overcome the potential for a conflict of interest, given they are also a "for-profit" provider. Currently, running such a system with limited industry involvement and therefore run by the physician or a non-device-related third party provider - is costing the patient, or physician, informally $\$ 100-\$ 400$ per patient per year, which is more than the reimbursement for the service (\$50-\$142 per patient device per year before tax). Charging patients directly may reduce enrolment and negate the benefits of remote monitoring. The dilemma is therefore whether we follow the guidelines and limit industry involvement, or use the government-funded system that industry is providing.

The collection, storage and distribution of remote monitoring information by industry are not clearly regulated. Regulation is complex, given all current industry providers have offshore servers and local distribution of data. While some companies have adopted a worldwide information security management system standard (ISO 27001), regulation has been company dependent and the current system is largely self-regulated by industry at the local level. This raises further issues and may exacerbate the potential for a conflict of interest regarding industry involvement. From a pragmatic perspective, current company involvement in remote monitoring is not particularly disproportionate to that in other areas of medicine such as electrophysiology. Most in-person device checks are currently facilitated by industry personnel and are indirectly funded by reimbursement from prosthesis sales and health insurance premiums. In addition, we routinely have an industry representative independently running 3D-mapping systems within the laboratory during complex patient procedures. The service provided by industry may facilitate utilisation of a system that improves patient outcomes and minimises the cost to patients and the health care system. ${ }^{8}$

Given the remoteness of a significant proportion of the Australian population in conjunction with the unique funding situation, an Australian heart rhythm consensus statement may help. A reasonable interim approach is likely to include full disclosure to the patient and documentation of any discussion and patient preference. It could also involve the offer of limited industry involvement, which would likely have some cost to the patient; industry-supported remote monitoring, which may reduce or negate the cost to the patient; or no remote monitoring at all. Therefore, in Australia, there is likely to be a deviation from the current guidelines with regard to industry involvement in remote monitoring, hopefully with the inherit motivation of improving patient care.

Competing interests: No relevant disclosures.

Provenance: Not commissioned; externally peer reviewed.

(c) 2017 AMPCo Pty Ltd. Produced with Elsevier B.V. All rights reserved.

References are available online at www.mja.com.au. 
1 Hindricks G, Taborsky M, Glikson M, et al. Implant-based multiparameter telemonitoring of patients with heart failure (IN-TIME): a randomised controlled trial. Lancet 2014; 384: 583-590.

2 Spencker S, Coban N, Koch L, et al. Potential role of home monitoring to reduce inappropriate shocks in implantable cardioverter-defibrillator patients due to lead failure. Europace 2009; 11: 483-488.

3 Burri $\mathrm{H}$, Sticherling $\mathrm{C}$, Wright $\mathrm{D}$, et al. Cost-consequence analysis of daily continuous remote monitoring of implantable cardiac defibrillator and resynchronization devices in the UK. Europace 2013; 15: 1601-1608.

4 Slotwiner D, Varma N, Akar JG, et al. HRS Expert Consensus Statement on remote interrogation and monitoring for cardiovascular implantable electronic devices. Heart Rhythm 2015; 12: e69-e100.
5 Lau CP, Zhang S. Remote monitoring of cardiac implantable devices in the Asia-Pacific. Europace 2013; 15 Suppl 1: i65-i68.

6 Australian Government Department of Health. Medicare Benefits Schedule book. Operating from 1 July 2016. Canberra: Commonwealth of Australia, 2015. http://www.health.gov.au/internet/mbsonline/publishing.nsf/ Content/45CBF3F97BA64FBDCA257FD5000E6666/\$File/201607-MBS.pdf (accessed Nov 2016).

7 Australian Government Department of Health. The prostheses list. http:// www.health.gov.au/internet/main/publishing.nsf/Content/healthprivatehealth-prostheseslist.htm (accessed Nov 2016).

8 Ricci RP, Vicentini A, D'Onofrio A, et al. Impact of in-clinic follow-up visits in patients with implantable cardioverter defibrillators: demographic and socioeconomic analysis of the TARIFF study population. J Interv Card Electrophysiol 2013; 38: 101-106. 